\title{
HIGHLIGHTS
}

MOVEMENT DISORDERS

\section{Image-based analysis reveals cause of parkinsonism}

The clinical features of idiopathic Parkinson disease (IPD), multiple system atrophy (MSA) and progressive supranuclear palsy (PSP) can overlap, making early diagnosis of these disorders a challenge for clinicians. Researchers have now developed an automated image-based classification procedure that can differentiate between these diseases. "This approach may help provide vital prognostic information to patients and their families," explains principal investigator David Eidelberg. "By reducing recruitment errors caused by misdiagnosed early-stage patients, our method can potentially improve the efficiency of clinical trials of diseasemodifying treatments."

IPD is the most common parkinsonian disorder and, like MSA and PSP, often manifests as tremor, rigidity and/or bradykinesia in the early stages of disease. These disorders, however, have markedly different prognoses and can vary considerably in their response to symptomatic therapies.

The automated diagnostic procedure used voxel-based spatial covariance analysis of ${ }^{18} \mathrm{~F}$-fluorodeoxyglucose-PET
4 ...our method can potentially improve the efficiency of clinical trials of disease-modifying treatments 77

brain images. Previous work by the researchers had shown that regional patterns generated from such analysis could differentiate patients with IPD, MSA or PSP from healthy individuals. Given the overlapping clinical features of IPD, MSA and PSP, the researchers used a multiple-pattern technique to discriminate the three disorders in their new study. "We computed the likelihood of each of the three possible diagnoses based on independent measurements of pattern expression in each subject's scan," explains Eidelberg. "An automated algorithm was then used to classify the individual patients according to the computed probability values." The accuracy of the system was determined by comparing the patients' algorithm-based classifications with their final clinical diagnoses, made on average 2.6 years after imaging. In a small number of patients, the automated result was compared with a diagnosis made at postmortem.

In total, 167 patients with parkinsonism of uncertain cause were assessed in this study. Following the final clinical diagnoses, the automated image-based system was found to have high specificity for all three disorders, ranging from $94 \%$ for PSP up to $97 \%$ for IPD. High sensitivity and predictive values for each disorder were also reported. "Our results are unique and illustrate the ability of automated pattern-based classification algorithms to provide an accurate and objective determination [of disease] in advance of definitive clinical diagnosis," says Eidelberg. "In the future, blinded, prospective imaging studies-ideally involving multiple sites, a larger validation cohort with repeat imaging, and moreextensive postmortem confirmation-will be needed to determine the accuracy of this automated categorization approach."

\section{Darran Yates}

Original article Tang, C. C. et al. Differential diagnosis of parkinsonism: a metabolic imaging study using pattern analysis. Lancet Neurol. 9, 149-158 (2010) 